\title{
Assessment by means of MRI Late Gadolinium Enhancement of periprocedural myonecrosis after main vessel stenting of coronary bifurcations
}

\author{
Aleksandra Michalek*, Dobrin Vassilev, Monika Odyniec, \\ Mariusz Furmanek, Tomasz Niemirka, Robert J Gil and Jerzy Walecki
}

Address: Mossakowski Medical Research Centre Polish Academy of Sciences, Warsaw, Poland

* Corresponding author

from 13th Annual SCMR Scientific Sessions

Phoenix, AZ, USA. 21 -24 January 2010

Published: 21 January 2010

Journal of Cardiovascular Magnetic Resonance 20 I0, I2(Suppl I):P58 doi: I0.I I86/I532-429X-I2-SI-P58

This abstract is available from: http://jcmr-online.com/content/I2/SI/P58

(C) 2010 Michalek et al; licensee BioMed Central Ltd.

\section{Introduction}

Delayed enhancement magnetic resonance imaging (LGE MRI) of the heart has a proven role for quantifying areas of myocardial injury. Percutaneous Coronary Intervention (PCI) of bifurcation lesions is considered as a high risk procedure. The factors governing periprocedural myonecrosis are not completely clear.

\section{Purpose}

The aim of this study was assessment and anatomical detection of myonecrosis by LGE MRI in the area supplied from the side branches of bifurcations treated by PCI.

\section{Methods}

Nineteen patients with stable coronary artery disease, with bifurcation stenosis were studied with LGE MRI (1.5 Tesla scanner) before $(24 \mathrm{~h})$ and after (72 h) stenting of bifurcation lesions. Angiographic measurements of the reference vessel diameters and side branch ostial diameters pre- and post-intervention were performed. Contrast enhanced images were obtained 12 to 18 minutes after i.v. gadolinium based contrast injection using breath-hold segmented gradient echo pulse sequence Myonecrosis was defined by new contrast hyperenhancement within target vessel perfusion territory

\section{Results}

After interventional procedure 11 (58\%) patients had evidence of new myocardial enhancement $(2.95 \pm 3.54$ grams, $2.78 \% \pm 4.64 \%$ of left ventricle mass). There was a correlation between LGE MRI and troponin I elevation pre- and post-procedure $(\mathrm{r}=.653, \mathrm{p}=.029$ and $\mathrm{r}=.785, \mathrm{p}$ $=.001)$, but not with pre- and post-interventional creatine - phosphokinase (CPK) and CPKMB. There was a significant difference in the diameter of the SB ostium after stent placement in patients with and without presence of new LGE MRI in the area supplied by SB (with LGE - .59 \pm .20 $\mathrm{mm}$ vs. without LGE $.99 \pm .47 \mathrm{~mm}, \mathrm{p}=.036)$. There was a significant correlation between ostial diameter post stenting and main vessel-side branch distal angle $(r=.565, \mathrm{p}=$ .018 ) and strong trend between this angle and myonecrosis assessed by LGE $(\mathrm{r}=-.435, \mathrm{p}=.081)$.

\section{Conclusion}

The post procedural myonecrosis detected by LGE MRI after bifurcation lesions stenting is associated with post stent implantation minimal lumen diameter at side branch ostium. The usual CPK and CPKMB tests were not able to detect adequately these changes. 